\title{
Characteristic numbers to describe the detail transfer quality of electro-chemical machining.
}

\section{J. Meijer* and J.C.M. Veringa**}

For reproducing processes such as electrochemical machining (ecm) the accuracy of the reproduction is an important process parameter.

Characteristic numbers which are a measure of this accuracy are proposed in this paper. Due to the non-linearity of the process a detail transfer function, which is helpful in linear cases, cannot be used, as will be shown from experimental work. Based on the same experiments, a characteristic radius and the standard deviation of a normal profile are proposed to describe the quality of the detail transfer. These numbers, which are easily interpreted criteria for quality and accuracy, have been proved to be almost independent of the geometry. Although developed for optimizing the ecm process, these values are also valid for edm and other reproducing processes. The usefulness of the characteristic numbers is illustrated by a comparison of continuous and pulsed ecm processes.

Keywords: electrochemical machining, process control, roughness (surface), transfer functions

Several authors reported improved reproduction accuracy with ecm by optimizing the process ${ }^{1-4}$. These results are unfortunately not easy to compare, because there is no standardized quality criterion. In other disciplines, such as optics, electronics or process control, transfer functions have proved to be very useful. Considering this, a harmonic frequency $v$ (which in this application can be thought of as a surface wave on the electrode) having an amplitude a(v). will generally cause an amplitude $a^{\prime}(v)$ on the surface of the product. From analogy with optical (contrast) transfer functions (ot $f)^{5}$ a surface reproduction can be described in the same way.

If there is no magnification or phase shift the transfer function can be simplified to $T(v)=a^{\prime}(v) / a(v)$. An example of a transfer function is given in Fig 1, starting at the value 1 at low frequencies and going to zero at high frequencies. The value where the amplitude reduction is $50 \%$ can be chosen as a main characteristic. In this special application the characteristic wavelength $\lambda_{50}=1 / \dot{v}_{50}$ is more relevant.

With linear processes it should be possible to measure a complete series of superposed frequencies in one test specimen. A simple to machine electrode profile, containing a series of harmonic frequencies, is a triangle. This can be expressed by

$$
y(x)=\sum_{k=0}^{\infty} \frac{4 H}{(2 k+1)^{2} \pi^{2}} \cos \left[2 \pi(2 k+1) \frac{x}{L}\right]
$$

Although the ecm process is not linear in itself, an attempt was made to find a quality characteristic in this way. To that end the corresponding $y^{\prime}(x)$ (after machining) was measured (Fig 2). Using Fourier transformation, the amplitudes $a^{\prime}(v)$ were found for the discrete frequencies $v=(k / L)$. Comparing this with the nominal amplitudes

- Twente University of Technology, Mechanical Engineoring Department, PO Box 217, 7500 AE Enschede, The Netherlands

- Now at IBM, Amsterdam which follow from $\mathrm{Eq}(1)$ the transfer function $\mathrm{T}(v)$ will be found. As already mentioned, this is only allowed when the process is linear, that means when the solution of a surface containing two (or more) superposed different harmonic frequencies gives the same result as a superposition of the individual solutions.

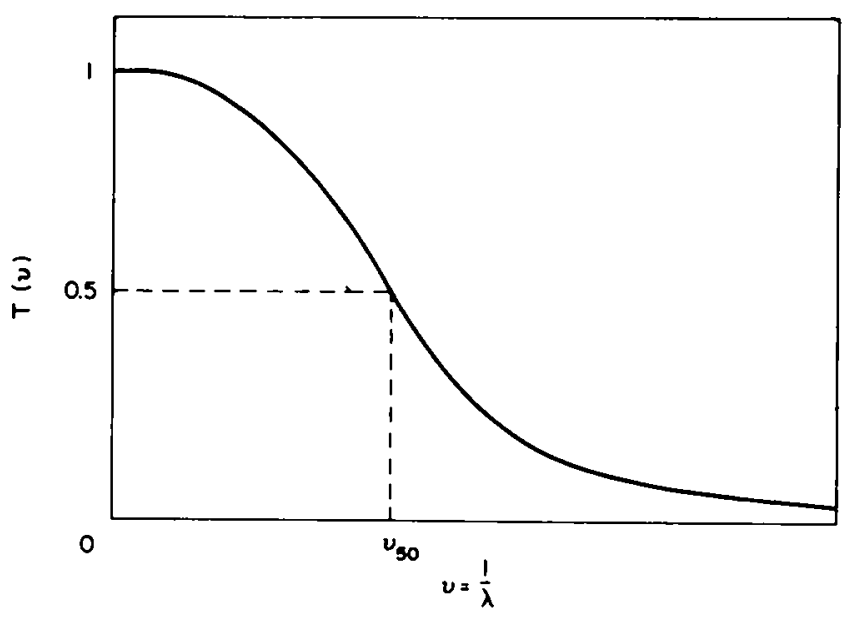

Fig 1 Detail transfer function

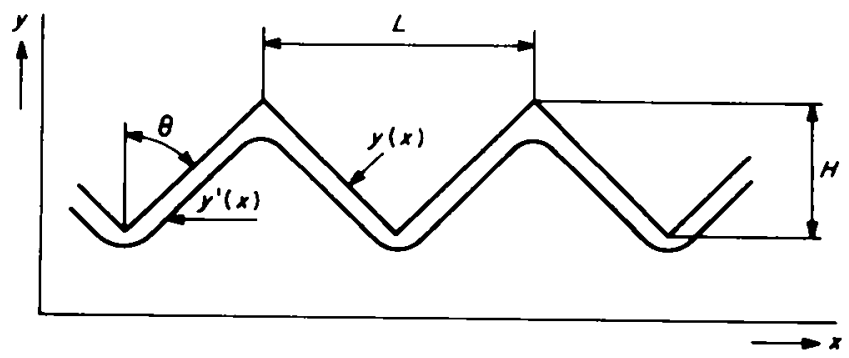

Fig 2 Triangular shaped electrode 


\section{Experimental arrangement}

In the experiment, two sets of workpieces were used. One set contained different electrode geometries (top-angles, sizes) and was used at constant process conditions to establish the influence of electrode geometry. The other set was used with varying process conditions at constant electrode geometry, to show that the model developed is useful to measure the detail transfer quality obtained at different process settings.

The triangular profile was situated at the top of a square electrode $(10 \times 10 \mathrm{~mm})$ which was driven transversely into the workpiece, composed of a package of strips. The profile ( $\mathrm{Fig} 3$ ) was digitized on a measuring machine at equidistant steps of $100 \mu \mathrm{m}$. The electrolyte flowed over the profile as shown in Fig 4.

\section{Results with $\lambda_{\text {so }}$ values}

Fig 5 shows the results for different electrode configurations, giving $\lambda_{50}$ values for constant process conditions. These values were found to be not independent of the electrode geometry ( $L$ as well as $\theta$ ), which makes the $\lambda_{\text {so }}$ value useless to define the process quality. From the experimental results it must be concluded that a complete detail transfer function cannot be found from copying one single profile containing a series of different frequencies.

This conclusion can be better understood by considering the ecm transfer of a single sine with a constant gap between both electrodes. As can be easily seen, the transferred profile is not an exact sine, so the condition of linearity is not fulfilled.

\section{The characteristic radius}

Nevertheless, the experimental data could be used to develop useful characteristics for assessing the quality of the detail transfer. As shown in Fig 2, the sharp edges of a triangular electrode will produce 'rounding' at the product, which is characteristic for ecm. From the measured data it could be concluded that $r_{b} \simeq 2 r_{t}$. The mean value $r_{c}=\left(r_{t}+r_{b}\right) / 2$ (the characteristic radius) has been proved to be reproducible for constant process conditions. The results, given in Fig 6 , are based on the same experimental data as used for Fig 5 . These results are not a function of the geometry, but only of the process conditions, and so offer a good measure for the process quality. Another advantage is that the characteristic radius has some importance for designers as well as for production engineers. Fig 7 shows how the product quality is influenced by changing the process conditions.

\section{Computing the results}

The computations have been based on least square methods, by which a normal profile is fitted through the measured points of the workpiece over a couple of periods. The 'norm-profile' is built-up of a triangle with top and bottom radii $r_{t}$ and $r_{b}$ respectively. When $r_{t}+r_{b}<L / \cos \theta$ (Fig 8 , right) then the profile is built-up of the radii only.

The free parameters in both figures are $r_{t}$ and $r_{b}$. In addition there are three linear parameters $\left(x_{0}, y_{0}\right.$ and $\left.\alpha\right)$ to fit the norm profile through the measured points (Fig 9 ). In this way the norm profile is determined by the function

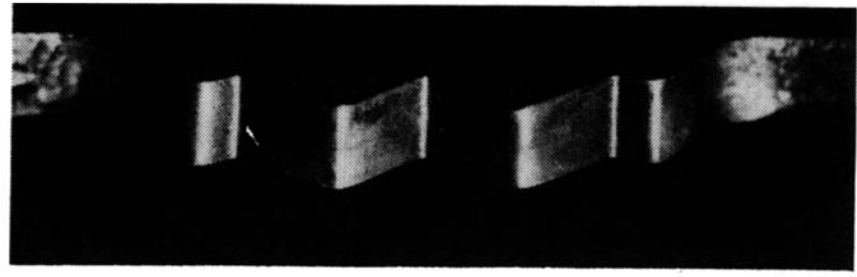

Fig 3 Workpiece

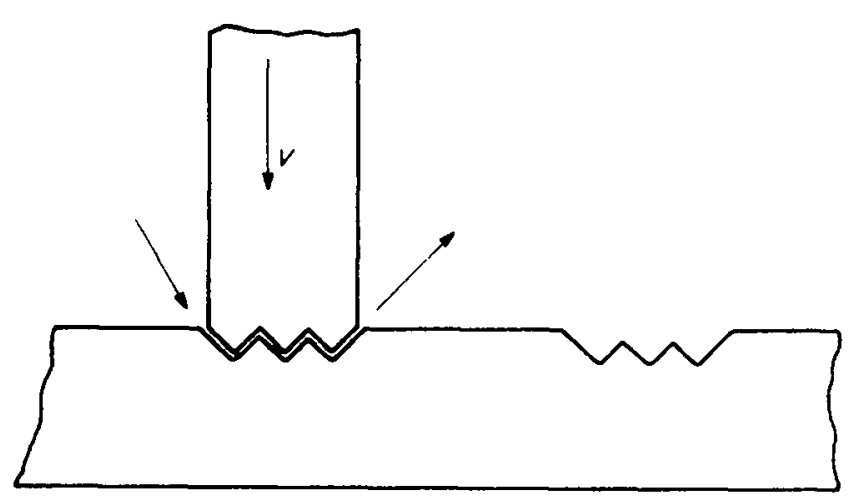

Fig 4 The eloctrode is driven into a package of strips

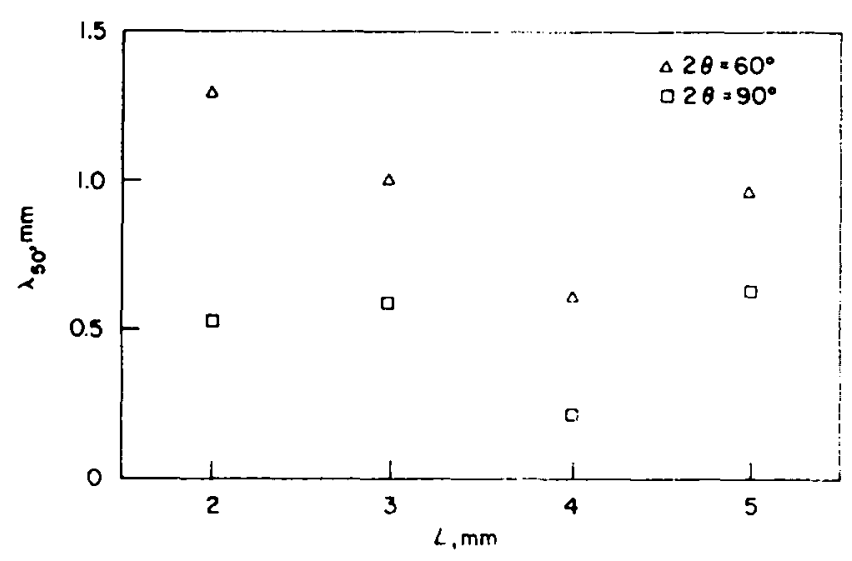

Fig $5 \lambda_{50}$-values: mild steel workpiece; copper electrode; voltage $=16 \mathrm{~V} ;$ electrolyte $=\mathrm{NaCl}, 12.5 \%, 40^{\circ} \mathrm{C} ;$ feed rate $=$ $2.9 \mathrm{~mm} / \mathrm{min}$

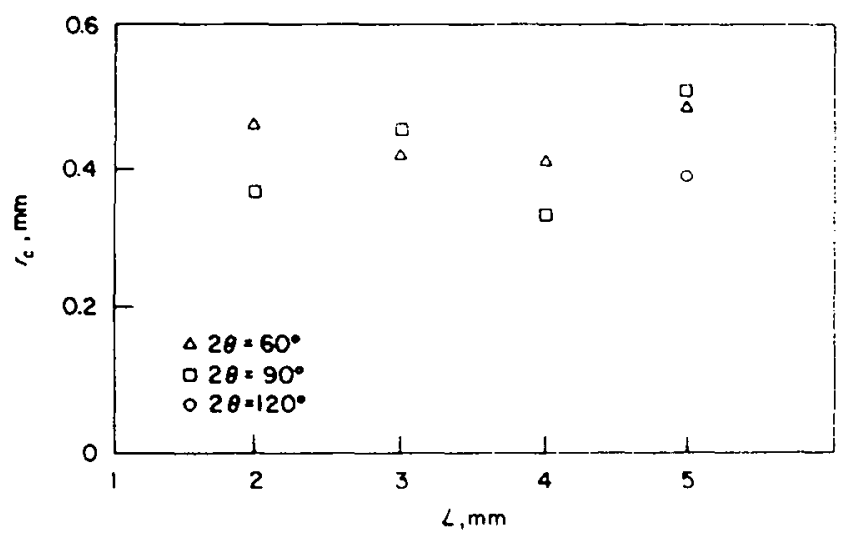

Fig 6 The characteristic radius which is not a function of the geometry 
$y(x)=y\left(x, x_{0}, y_{0}, \alpha, r_{t}, r_{b}\right)$. To obtain the best fitting with the measured points ${ }^{6}$ the function $F\left(x_{0}, Y_{0}, \alpha, r_{t}, r_{b}\right)=\Sigma\left[y(x)-y^{\prime}\right.$ $(x)]^{2}$ should be minimized with respect to all five parameters. For this purpose a standard procedure ${ }^{7}$ has been used to give a fast solution. The accuracy of curve fitting is given by the standard deviation:

$$
\sigma=\frac{\left(\sum\left[y(x)-y^{\prime}(x)\right]^{2}\right)^{1 / 2}}{n-5}
$$

This value is a direct measure of the surface quality of the profile. For the cases shown in Fig 7 a standard deviation $\sigma \simeq 0.07 \mu \mathrm{m}$ was found.

\section{Pulsed ecm}

One of the aims in using a pulsed mode of ecm is to obtain a better reproduction quality. When machining in the pulsed mode (at the same conditions) it is surprising to find no significant change of the characteristic radii (note that for a fair comparison the machining velocity was selected in accordance with the duty cycle). Nevertheless, pulsed ecm gives a much better surface (Fig 10). The difference between both surfaces is clearly shown by the second characteristic, the standard deviation of the fitting (Eq (2)). In the continuous mode $\sigma=0.07 \mu \mathrm{m}$ has been found, while in the pulsed mode $\sigma=0.02 \mu \mathrm{m}$ has been reached. In the
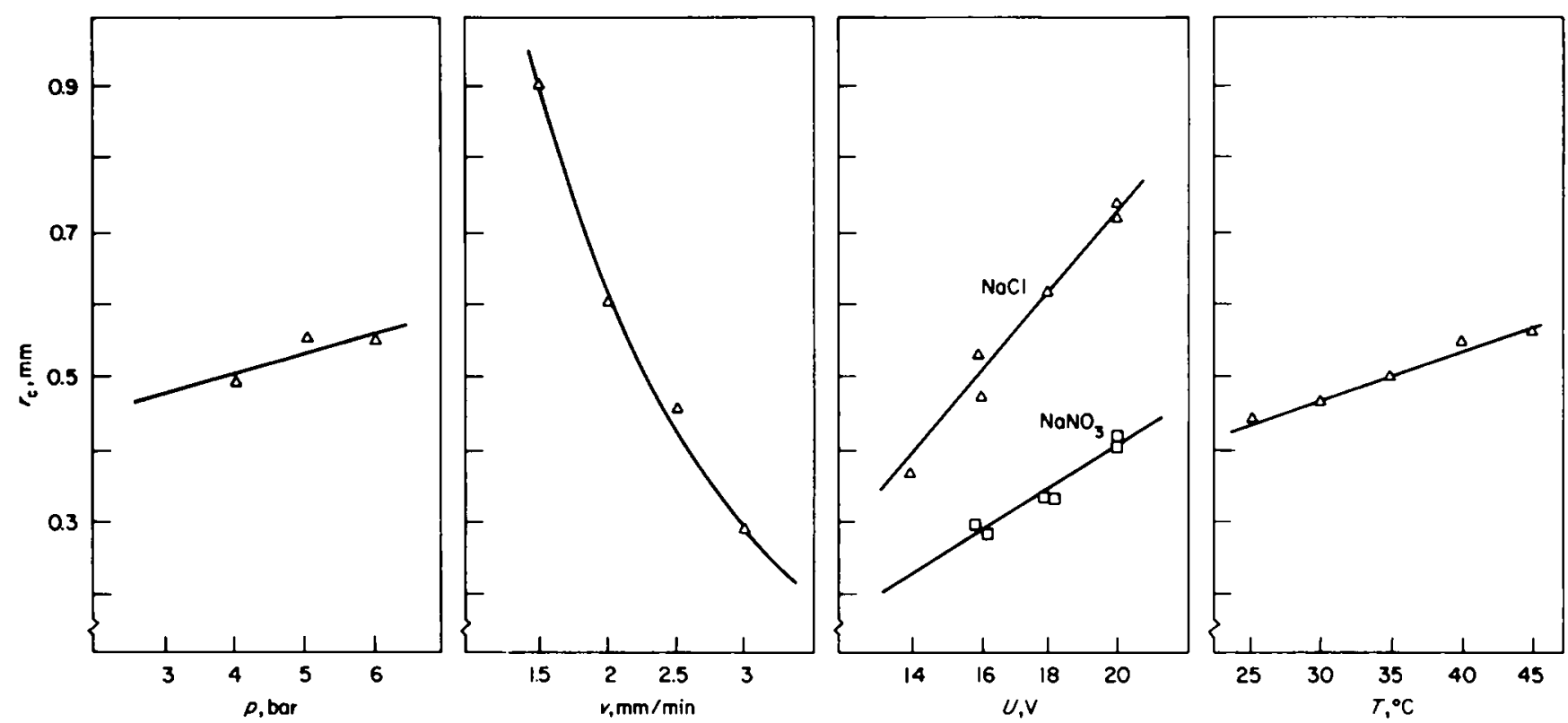

Fig 7 Influence of process conditions on the characteristic radius. Standard deviation of the given points $=0.015 \mathrm{~mm}$

\begin{tabular}{|ll|}
\hline Nomenclature \\
$H$ & height of a triangle \\
$L$ & base length of triangle \\
$T$ & transfer function \\
$T$ & temperature \\
$U$ & electrical potential \\
$a$ & amplitude of electrode \\
$a^{\prime}$ & amplitude after transfer \\
$n$ & number of points \\
$p$ & pressure \\
$r_{t}$ & top radius \\
$r_{\mathrm{b}}$ & bottom radius \\
$r_{\mathrm{c}}$ & characteristic radius \\
$V$ & velocity \\
$x$ & ordinate \\
$V$ & height ordinate \\
$V_{(x)}$ & norm function \\
$y^{\prime}(x)$ & measured ordinates \\
$\alpha$ & angle \\
$2 \theta$ & top angle of triangle \\
$v$ & frequency \\
& \\
\hline
\end{tabular}

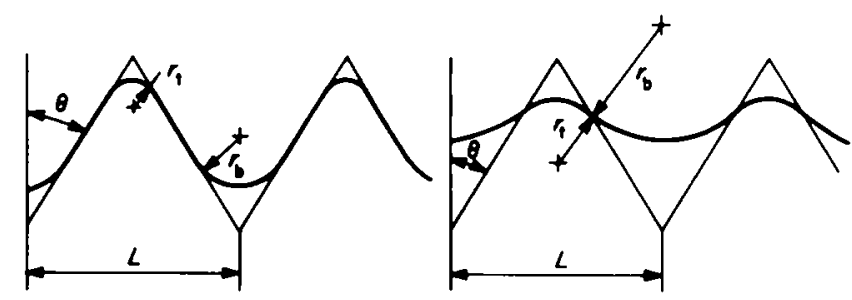

Fig 8 Norm-profiles

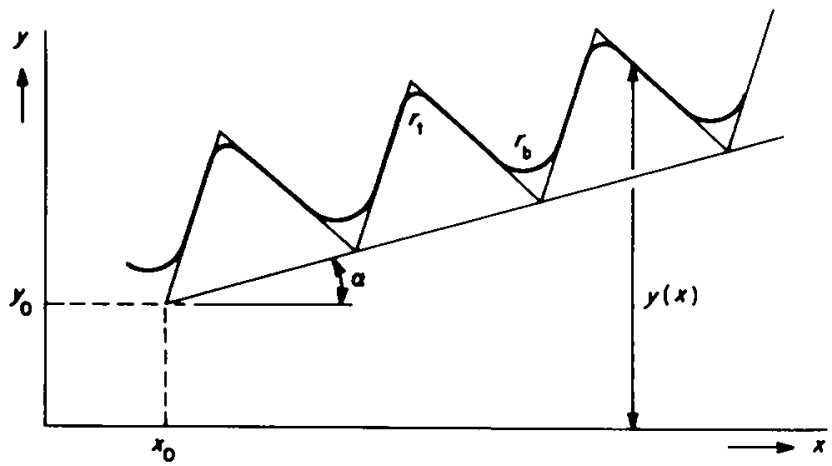

Fig 9 The norm-profile in the $x-y$ plane (measurement co-ordingtes) 
latter case all stream lines on the machined surface were removed.

\section{Conclusions}

The characteristic radius $r_{c}$ has been shown to be a helpful number to describe the quality of the detail transfer of ecm or similar shape reproducing processes. This value is only a function of the process parameters and independent of the geometry. In terms of product design, this value can be interpreted as a real radius.

The overall accuracy of the reproduction is given by the standard deviation $\sigma$ of the curve fitting. This value, which is considerably improved by pulsed machining, can be interpreted as surface roughness (rms-value). This makes both values useful to both designers and production engineers.

\section{References}

1 Kozak J., Lubkowski K., Debrowki L. and Steczowitcz M. Study on the influence of process parameters on the technological effects of pulse ECM. Proc. ISEM 6, Cracow, June 1980, 233-238

2 Alieksiejor G.A., Lapides L.M. and Moroz I.I. Methods of increasing accuracy in ECM forming. Proc. ISEM 6. Crocow, June 1980, 281-284

3 Könis W., Neubauer J. and Lindentauf H.P. Zur Ausbildungund Wiederholgenauigkeit bei Elektrochemisem Senken. TZ fuer Motallbearbeitung 74, 1980

4 Yu C.S., Yang J.S. and Cheng C.K. The relation between copying accuracy and electrolytes of ECM for titanium alloys. CIRP Annals, 1981, 30/1), 123-127

5 Ditchburn R.W. Light. Academic Press, London, 1976

6 Mojer J. and de Bruin W. Determination of flatness from straightness measurements and characterization of the surface by four parameters. Procision Enginouring, Jon. 1981, 3(1), 17-22

7 NAG-Algol 60 Library. Numerical Algorithms group, 1981
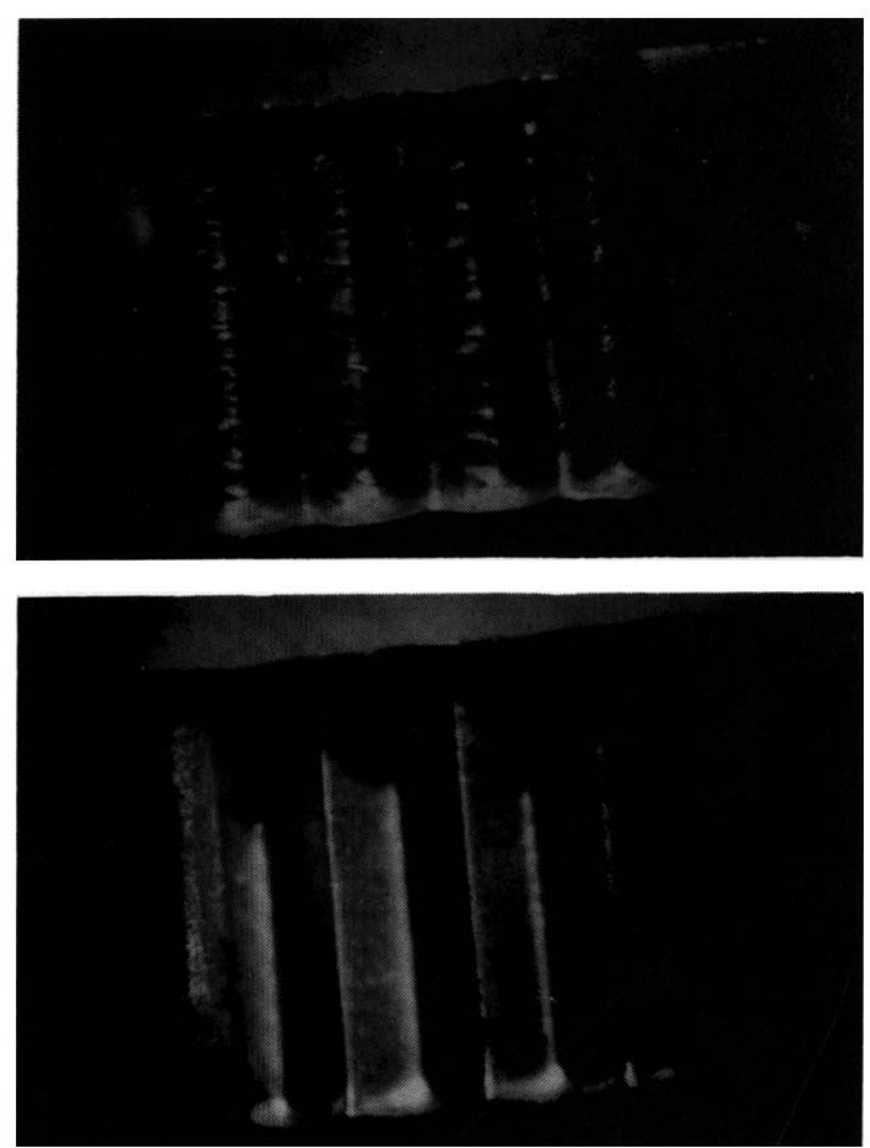

Fig 10 Mild steel machined at $16 \mathrm{~V}, 6$ bar, $\mathrm{NaClO}_{3} 25 \%$, $\mathrm{T}=25^{\circ} \mathrm{C}$. At the top; continuous process $\mathrm{v}=2.25 \mathrm{~mm} / \mathrm{min}$. At the bottom; pulsed process at $200 \mathrm{~Hz}$, pulse time $1 \mathrm{~ms}$, $v=0.45 \mathrm{~mm} / \mathrm{min}$ 